\title{
Cualidades de profesores en matemática recordados como buenos por futuros profesores en matemática
}

Natalia Sgreccia, Mariela Cirelli y María-Beatriz Vital

\author{
Basta un profesor —iuno solo! — para \\ salvarnos de nosotros mismos $y$ \\ hacernos olvidar a todos los demás
}

(Pennac, 2008: 147)

\section{RESUMEN}

En este artículo se analizan las cualidades de docentes en Matemática recordados como buenos profesores por ingresantes universitarios a la carrera Profesorado en Matemática de la Universidad Nacional de Rosario (Argentina), en el marco de una actividad en la que se les solicita recordar las cualidades de dos de los mejores docentes de toda su escolaridad. Se las compara con cualidades de "buenos docentes" en matemática con el objeto de analizar la ponderación de ciertos rasgos en los profesores de esta disciplina con respecto a profesores de cualquier otra en aspirantes a profesor en Matemática. Metodológicamente se considera un gran cúmulo de datos para realizar interpretaciones cualitativas mediante la técnica de análisis de contenido. Se encuentran muchas semejanzas entre los docentes memorables del estudio, independientemente de la disciplina, así como también algunos rasgos distintivos de los profesores de la especialidad Matemática. Se subraya la importancia de la biografía escolar para la construcción del conocimiento profesional docente, particularizando en el Profesorado en Matemática.

Palabras clave: biografía, profesor, matemática, escuela secundaria, Argentina.

Natalia Sgreccia

sgreccia@fceia.unr.edu.ar Argentina. Doctora en Humanidades y Artes con mención en Ciencias de la Educación, Universidad Nacional de Rosario, Argentina; Magíster en Didácticas Específicas con mención en el área Matemática, Universidad Nacional del Litoral, Argentina. Profesora Asociada Dedicación Exclusiva en el Departamento de Matemática de la Escuela de Ciencias Exactas y Naturales, Facultad de Ciencias Exactas, Ingeniería y Agrimensura, Universidad Nacional de Rosario. Temas de investigación: formación de profesores en matemática, didáctica de la geometría, recursos didácticos y educación a distancia.

\section{Mariela Cirelli}

cirelli@fceia.unr.edu.ar Argentina. Licenciada en Matemática, Universidad Nacional de Rosario, Argentina; Profesora de Enseñanza Media y Superior en Matemática, Universidad Nacional de Rosario, Argentina. Profesora Adjunta Dedicación Exclusiva en el Departamento de Matemática de la Escuela de Ciencias Exactas y Naturales de la Facultad de Ciencias Exactas, Ingeniería y Agrimensura, Universidad Nacional de Rosario. Temas de investigación: formación de profesores en Matemática.

\section{María Beatriz Vital}

vital@fceia.unr.edu.ar Argentina. Licenciada en Matemática, Universidad Nacional de Rosario, Argentina; Profesora de Enseñanza Media y Superior en Matemática, Universidad Nacional de Rosario, Argentina. Profesora Adjunta Dedicación Semiexclusiva en el Departamento de Matemática de la Escuela de Ciencias Exactas y Naturales, y en el Departamento de Matemática de la Escuela de Formación Básica de la Facultad de Ciencias Exactas, Ingeniería y Agrimensura, Universidad Nacional de Rosario. Temas de investigación: formación de profesores en Matemática. 


\title{
Qualidades de professores de matemática lembrados como bons por futuros professores de matemática
}

\section{RESUMO}

Neste artigo se analisam as qualidades de docentes de matemática lembrados como bons professores por formandos universitários do curso Professorado em Matemática da Universidade Nacional de Rosário (Argentina), no marco de uma atividade na que se solicita recordar a dois dos melhores docentes de toda sua escolaridade. As respostas são comparadas com qualidades de "bons docentes" em general (de qualquer disciplina) com o objetivo de analisar a ponderação de certas características nos professores desta disciplina com respeito a professores de qualquer outra matéria, segundo aspirantes a professores de matemática. Metodologicamente se considera um grande acúmulo de dados para realizar interpretações qualitativas mediante a técnica de análise de conteúdo. Se encontram muitas semelhanças entre os docentes memoráveis do estudo, independentemente da disciplina, assim como também algumas características distintivos dos professores da especialidade matemática. Se ressalta a importância da biografia escolar para a construção do conhecimento profissional docente, particularizando no Professorado de Matemática.

Palavras chave: biografia, professor, matemática, escola secundaria, Argentina.

\section{Qualities of Mathematics teachers that had a positive impact for future Mathematics teachers}

\begin{abstract}
This article analyzes the qualities of teachers in mathematics that were remembered as good teachers by young university students who just enrolled for the degree in Teachers in Mathematics at the National University of Rosario (Argentina), in the framework of an activity in which they are asked to remember two of the best teachers of their entire schooling. The obtained results are compared with qualities of "good teachers" in general (of any discipline) in order to analyze the relevance of certain features in the teachers of Mathematics compared with the teachers of any other discipline, according to candidates for teachers in Mathematics. Methodologically, it was possible to take into account a great accumulation of data in order to make qualitative interpretations through the technique of content analysis. There are many similarities among the memorable teachers of the study, regardless of discipline, as well as some distinctive features of the teachers of Mathematics. The authors point out the importance of the school biography for the construction of the teacher's professional knowledge, particularizing in the Professorship in Mathematics.
\end{abstract}

Key words: biography, teacher, mathematics, secondary school, Argentina.

Recepción: 30/09/17. Aprobación: 15/01/18. 


\section{Presentación y antecedentes sobre el tema}

Este artículo se enmarca en parte del trabajo que se viene realizando en el Proyecto de Investigación "Procesos de acompañamiento en la formación inicial y continua de profesores en Matemática" (ING445, 2014-2017) que, en términos generales, pretende analizar los procesos de formación inicial y continua del conocimiento profesional docente de profesores en Matemática, procurando echar luz acerca de cómo se le puede favorecer desde la formación sistemática.

Sin duda la biografía escolar constituye un potente dispositivo para ello (Alliaud, 2004). De allí que no sólo lo hayamos ido enfatizando en nuestras tareas docentes, sino que, y sobre todo en lo concerniente a lo que aquí presentamos, nos hemos ido adentrando en investigaciones sobre el tema.

Cabe advertir que nuestras tareas de docencia e investigación están radicadas en la Facultad de Ciencias Exactas, Ingeniería y Agrimensura de la Universidad Nacional de Rosario (UNR), puntualmente en la carrera Profesorado en Matemática (PM).
Tomamos como punto de partida la primera actividad que los ingresantes al PM realizan en la asignatura Práctica de la Enseñanza I, de primer año. La misma consiste en identificar a los dos mejores profesores que hayan tenido en su trayectoria escolar especificando, entre otras cuestiones, tres características positivas por las cuales los eligieron. Lo escriben en una ficha impresa en papel (figura 1), con tamaño intencionalmente pequeño (8cm x $6 \mathrm{~cm})$. De este modo, podríamos decir que cada docente memorable queda caracterizado por una terna de cualidades.

Nuestro primer trabajo (Sgreccia y Cirelli, 2015) consistió en recolectar esas características positivas, "cualidades" en lo que sigue, en los 13 años comprendidos hasta ese entonces (2002-2014). Fueron 3 701 las cualidades mencionadas por los estudiantes, siendo 566 distintas entre sí. A los efectos de la investigación se procesaron de acuerdo a las cinco subpreguntas nodales señaladas por Bain (2007) cuando intenta dilucidar qué hacen "los mejores profesores".

Figura 1. Modelo de ficha que completan los ingresantes al PM

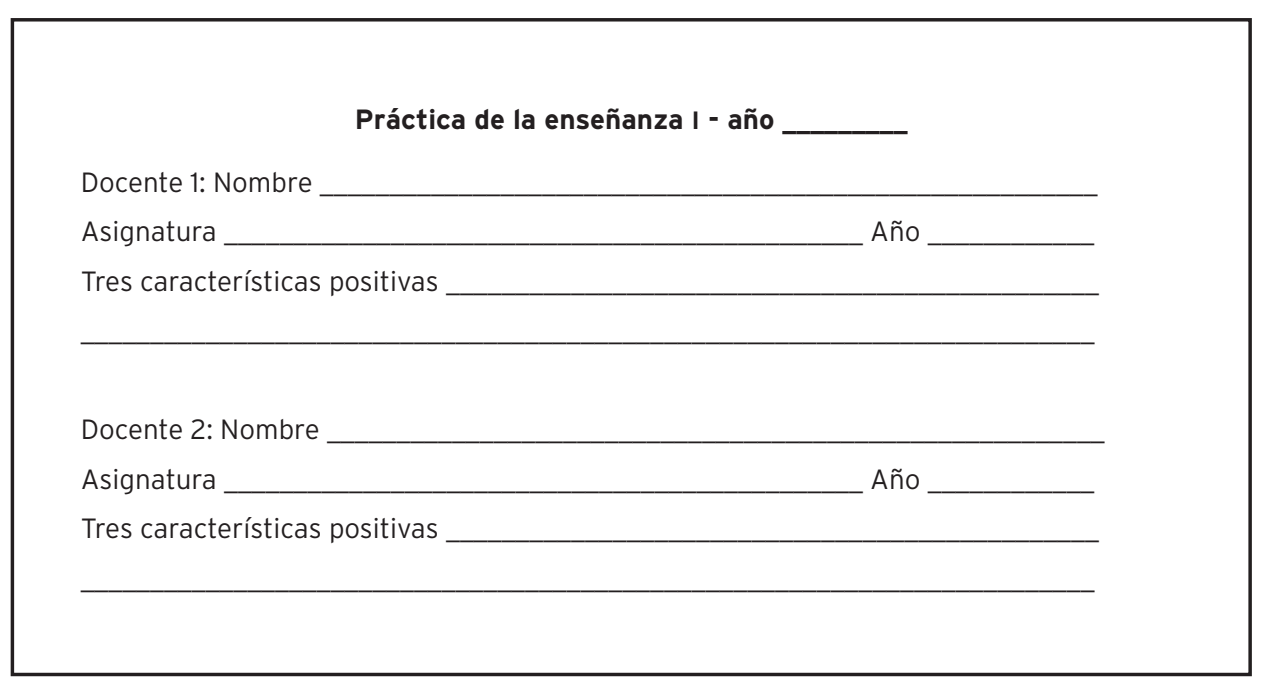


Puntualmente, en nuestro caso, hallamos las siguientes dimensiones:

1. ¿Qué motiva a un alumno?.' que el profesor disfrute de su tarea; que la materia le genere interés; que el profesor esté motivado; que se capte su atención; que sea invitado a dar lo mejor de sí; que aprenda de manera significativa; que el profesor sea un profesional comprometido.

2. ¿Cómo preparan las clases?: con conocimiento; con responsabilidad; con dedicación; con eficiencia; procurando la accesibilidad del conocimiento; de manera diferente; para contribuir al pensamiento de los alumnos; con actividades vinculadas a la realidad; con actividades y materiales para el estudio.

3. ¿Cómo dirigen las clases?: explicando y enseñando bien; destinando tiempo para el aprendizaje; siendo claros; siendo concisos; propiciando un ritmo adecuado; expresándose de manera adecuada; propiciando un clima agradable; propiciando herramientas transversales; promoviendo la participación estudiantil; con presencia imponente.

4. ¿Cómo tratan a sus alumnos?: con amabilidad y gracia; con comprensión; con calidad humana; con compañerismo y demostrando que sus alumnos les importan; promoviendo respeto y disciplina; con alegría; de manera estricta; con generosidad; con apertura; estableciendo límites claros; con afecto; de manera personalizada; con sinceridad; siendo carismáticos; siendo flexibles; siendo humanos.

5. ¿Cómo evalúan?: siendo exigentes; siendo equitativos; dando oportunidades; de manera continua; mediante ciertos modos valorados.
Hubo seis cualidades, de las 566 identificadas, que sobresalieron con respecto al resto por su marcada aparición. Ellas son: explica bien, exigente, paciente, buena persona, claro, comprensivo. Recordando, además, que cada docente memorable está conformado por una terna de cualidades, interesó conocer qué características positivas acompañan a cada una de las sobresalientes (Sgreccia, Cirelli y Vital, 2015). Esto con la intención de reconocer ciertos rasgos especialmente ponderados, a modo de tipología, que indiquen las cualidades sobresalientes con sus respectivas dos cualidades recurrentes. Se desprendió una terna casi inseparable: exigente-explica bien-paciente. De este modo, la cualidad exigente se destaca en cada terna que conforma la tipología, advirtiendo que se trata de una exigencia basada en lo que el docente les brinda en cada encuentro a través de sus buenas explicaciones y con mucha paciencia.

Paralelamente, se procuró sistematizar las tareas de enseñanza que se vienen desarrollando en el PM vinculadas de manera explícita con la biografía escolar (Vital, Sgreccia y Cirelli, 2015). Resultó que se constituye en tema objeto de estudio en todos los años de la carrera: Práctica de la Enseñanza I (primer año), Pedagogía (segundo año), Práctica de la Enseñanza II (tercer año) y Residencia (cuarto año). Las actividades procuran hacer rememorar experiencias significativas vividas por los estudiantes durante toda su trayectoria escolar, ya que "las pedagogías del contar alientan una narrativa vital de maneras y formas de enseñar y de aprender que aportan a la comprensión del desarrollo de la identidad profesional y de los procesos de enseñanza y los de aprendizaje dentro y fuera de las aulas" (Sarasa, 2014: 159). También fue posible advertir el robustecimiento, en el sentido de consolidación y fortificación, de las actividades a través de los años.

\footnotetext{
${ }^{1}$ Cabe advertir que en esta ocasión se acompaña cada dimensión por "familias" de cualidades, que procuran agrupar características positivas relativamente análogas a nivel semántico señaladas por los estudiantes.
} 
Con el fin de complementar tales estudios y asumiendo a la indagación autobiográfica como una reconfiguración del pasado que otorga sentido a las circunstancias presentes, se realizó un grupo enfocado con cuatro egresadas relativamente recientes de la carrera que ya han transitado sus primeros cinco años de socialización profesional (Vital, Sgreccia y Cirelli, 2016). Por un lado, las egresadas explicitaron los sentidos atribuidos a las cualidades de los docentes que habían indicado como memorables cuando iniciaron la carrera (figura 1). Por otro lado, compararon dichas cualidades con las que creen poseer como profesoras en Matemática en la actualidad. Resultó que las cualidades reconocidas en ellas son prácticamente las mismas que las atribuidas a sus docentes memorables.

A través de los estudios fue emergiendo la inquietud acerca de las eventuales peculiaridades que pudieran presentar entre esos docentes memorables los que en particular son de Matemática. Esto fundamentado en que se trata de una carrera de Profesorado de esta disciplina y que la importancia del contenido es insoslayable. Se considera a tal un tema de agenda, pues el estado de conocimiento sobre el tema construido sobre la base de otras investigaciones (tales como Álvarez et al., 2011; Osorio, 2012; De Laurentis, 2014) indica una vacancia tanto en cuanto a la especificidad de la disciplina en el estudio de docentes memorables como en lo referido al nivel secundario de educación (la mayoría analiza docentes del nivel universitario).

Concretamente en este trabajo, que se basa predominantemente en "buenos docentes" del nivel secundario de educación, focalizamos la atención en responder: ¿Cómo se vincula el conjunto total de cualidades que aquí informamos, referidas a docentes de diversas asignaturas, con las particularmente señaladas por los docentes memorables que se desempeñaban en la asignatura Matemática?

Cabe advertir que se considera como supuesto de partida que las seis cualidades sobresalientes (explica bien, exigente, paciente, buena persona, claro, comprensivo) de los profesores en general están aún más marcadas en los profesores en Matemática.

\section{Marco teórico-metodológico}

El enfoque del estudio es predominantemente cualitativo, dado que si bien se vale de un cúmulo muy importante de datos, busca interpretarlos para comprender el fenómeno de la buena enseñanza con la mayor profundidad posible desde este caso en particular: ingresantes al PM, sin ánimos de generalización (Hernández et al., 2006).

El alcance es descriptivo-comparativo-interpretativo, dado que se caracterizan las cualidades recogidas de los buenos docentes, se comparan las de los de Matemática con las de docentes de otras disciplinas y se producen algunas posibles interpretaciones por parte del equipo de investigación.

El estudio se nutre de una perspectiva basada en la opinión de los estudiantes, la cual se considera relevante por las numerosas experiencias de los alumnos con diversos profesores (Malikow, 2005-2006). En particular, aquí involucra a egresados recientes de la escuela secundaria en relación con sus experiencias como alumnos de buenos docentes por ellos especialmente recordados.

Los participantes de la investigación son 716 alumnos ingresantes al PM que cursaron la asignatura Práctica de la Enseñanza I de primer año entre los años 2002 y 2016 inclusive (es decir, las cohortes correspondientes a 15 años consecutivos). El diseño de la investigación es no experimental, debido a que los grupos de participantes ya estaban constituidos, independientemente de este estudio, desarrollándose la investigación en un ámbito natural de cursado de una cátedra universitaria. Los estudiantes provienen, en cantidades relativamente similares, tanto de la ciudad de Rosario (de diversas escuelas secundarias) como de otras localidades de la provincia de Santa Fe (donde se ubica Rosario) y de otras provincias aledañas. 
Para el procesamiento de la información se empleó la técnica de análisis de contenido (Cabrera, 2009) en tres fases:

- Fase 1. Transcripción de cada cualidad a una matriz de datos Excel (tabla 1) donde se especifica la dimensión de referencia (en términos de Bain, 2007) y la frecuencia absoluta de aparición de cada cualidad.

Las cualidades, expresadas en la forma en que lo hicieron los aspirantes a profesor, se constituyen en las modalidades del estudio. De este modo cada dimensión queda conformada por una cierta cantidad de modalidades (cualidades distintas entre sí): 73 en la dimensión 1 (n1=73), n2=151, n3=156, n4=158 y n5=46.

- Fase 2. Agrupamientos de las modalidades según focos conceptuales definidos por las investigadoras luego de la inmersión en los datos. Estos agrupamientos resultaron ser lo que hemos denominado "las familias" emergentes en el estudio. Así, la cantidad de familias de cualidades en cada dimensión, de 1 a 5, son: 7, 9, 10,16 y 5 , respectivamente.
- Fase 3. Procedimiento análogo al de la Fase 1 pero aplicado sólo a los docentes de Matemática.

El nivel educativo altamente predominante en el que se desempeñan los docentes reconocidos como buenos, tal como se solicitó en la consigna de la actividad, corresponde al secundario. En cuanto al sexo, el 70\% es femenino. Acerca de las asignaturas, predomina holgadamente Matemática (595 sobre 1418 en total).

Las dimensiones de análisis han sido delimitadas por las cinco perspectivas señaladas por Bain (2007), como resultado de su extensa investigación de 15 años en distintas universidades de Estados Unidos. Este investigador procuró encontrar rasgos comunes entre cientos de profesores cuyo trabajo había sido considerado excepcional, tanto por sus alumnos, así como por sus colegas y las instituciones en las que se desempeñaban. Realizó su estudio en una variedad de universidades y de disciplinas, procurando cierta representatividad.

En esta ocasión se comparan los datos (profesores en Matemática/profesores en otras asignaturas) desde tres planos:

Tabla 1. Matriz de datos empleada para el procesamiento

\begin{tabular}{|c|c|c|c|c|}
\hline \multirow{2}{*}{ Frecuencia } & Dimensión 1 & $\ldots$ & Frecuencia & Dimensión 5 \\
\hline$\ldots$ & Modalidad Dim1-1 & & $\ldots$ & Modalidad Dim5-1 \\
\hline$\ldots$ & Modalidad Dim1-2 & & $\ldots$ & Modalidad Dim5-2 \\
\hline$\ldots$ & $\ldots$ & & $\ldots$ & $\ldots$ \\
\hline$\ldots$ & Modalidad Dim1-n1 & & $\ldots$ & Modalidad Dim5-n5 \\
\hline
\end{tabular}


- Con respecto a las dimensiones de análisis.

- Con respecto a las familias emergentes en cada dimensión.

- Con respecto a ciertas cualidades distinguidas (las tres primeras por dimensión).

\section{Resultados}

Se han registrado 4302 cualidades, de las cuales 585 son distintas entre sí (en 78 ocasiones se menciona "buen profesor", sin ubicarlo en dimensión alguna). De ellas, 1815 cualidades corresponden a los profesores en Matemática, siendo 321 distintas entre sí (33 “buen profesor”). Es decir, el 42.19\% de las cualidades mencionadas por los estudiantes corresponde a características de profesores en Matemática, obteniéndose este mismo porcentaje si la cualidad "buen profesor" no es tenida en cuenta. A continuación, se presentan los hallazgos de acuerdo con los tres planos de interés.

\section{Con respecto a las dimensiones de análisis}

En la tabla 2 se presenta la frecuencia de cualidades en cada dimensión, tanto para los registros relativos a profesores en Matemática como de otras disciplinas (por ejemplo, 118 y 171 respectivamente para la dimensión 1). Se la compara, a su vez, con respecto a la frecuencia total de cualidades (1 782 y 2 442, respectivamente). Esto con el propósito de conocer comparativamente la distribución de cualidades por dimensión.

A partir de lo consignado en la tabla 2, se pueden advertir tendencias relativamente similares en los profesores en Matemática y de otras disciplinas, sobre todo en tres de las dimensiones (1, 2 y 5). La mayor diferencia se presenta en las dimensiones 3 (ciómo dirigen las clases?') y 4 (¿Cómo tratan a los alumnos?') ponderándose la primera más en los profesores en Matemática y la segunda en los de otras disciplinas. Esto denota la importancia de la presencia activa del profesor en Matemática en el momento de la clase.

Tabla 2. Frecuencia de cualidades en cada dimensión con respecto a la frecuencia total de cualidades ${ }^{2}$

\begin{tabular}{|c|c|c|}
\hline \multirow{2}{*}{ Dimensión } & Matemática & Otras disciplinas \\
\hline 1 & $118 / 1782=0.0662$ & $171 / 2442=0.0700$ \\
\hline 2 & $269 / 1782=0.1510$ & $392 / 2442=0.1605$ \\
\hline 3 & $654 / 1782=0.3670$ & $736 / 2442=0.3014$ \\
\hline 4 & $589 / 1782=0.3305$ & $895 / 2442=0.3665$ \\
\hline 5 & $152 / 1782=0.0853$ & $248 / 2442=0.1016$ \\
\hline
\end{tabular}

\footnotetext{
${ }^{2}$ En la frecuencia total de cualidades se restó, tanto en los profesores en Matemática como en otras disciplinas, la cantidad relativa a "buen profesor" en general (1 815-33 y 4 302-78, respectivamente).
} 
En la tabla 3 se muestra, tanto para profesores en Matemática como de otras disciplinas, la cantidad de cualidades distintas, es decir de modalidades, para cada una de las cinco dimensiones de análisis. Se efectúa con la intención de observar el grado de variabilidad de las cualidades mencionadas dentro de cada dimensión en términos comparativos entre los profesores en cuestión (en Matemática y de otras disciplinas).

Como se desprende de la tabla 3, en todas las dimensiones, excepto en la quinta, se observa una mayor variedad de cualidades distintas asociadas con docentes en Matemática que con profesores de otras disciplinas. Particularmente esta variedad se ve acentuada en la dimensión 1 (¿Qué motiva a un alumno?), siguiéndole la 4 (¿Cómo tratan a sus alumnos?). Tal variedad parece indicar que hay un espectro relativamente amplio de componentes del accionar del profesor en Matemática que son apreciadas por sus estudiantes.

\section{Con respecto a las familias emergentes en cada dimensión}

En las tablas 4 a 8 se recorren las familias emergentes por cada dimensión de análisis. Se acompañan de algunos extractos de cualidades mencionadas por los estudiantes para ilustrar las ideas allí contenidas. Para cada una se consignan las frecuencias respectivas registradas en los profesores en Matemática, así como de otras disciplinas, en comparación con la frecuencia total de la dimensión (por ejemplo, en la dimensión 1, 118 para profesores en Matemática y 171 para los de otras disciplinas). Finalmente, se explicitan las diferencias entre las frecuencias relativas de cada familia presentes en los profesores en Matemática y de otras disciplinas.

En la dimensión 1, ¿Qué motiva a un alumno? (tabla 4), no se observan diferencias notables en las distribuciones de frecuencias relativas de las distintas familias de cualidades correspondientes a los docentes en Matemática y a los docentes de otras disciplinas.

Tabla 3. Cantidad de modalidades (cualidades distintas) en cada dimensión sobre la frecuencia total de cada dimensión

\begin{tabular}{|c|c|c|}
\hline Dimensión & Matemática & Otras disciplinas \\
\hline 1 & $48 / 118=0.4068$ & $25 / 171=0,1462$ \\
\hline 2 & $68 / 269=0.2528$ & $83 / 392=0,2117$ \\
\hline 3 & $88 / 654=0.1346$ & $68 / 736=0,0924$ \\
\hline 4 & $102 / 589=0.1732$ & $56 / 895=0,0626$ \\
\hline 5 & $14 / 152=0.0921$ & $32 / 248=0,1290$ \\
\hline
\end{tabular}


Tabla 4. Comparación de cantidades por familias en la dimensión 1: ¿Qué motiva a un alumno?

\begin{tabular}{|c|c|c|c|c|}
\hline Familias $^{3}$ & Extractos de cualidades mencionadas & Matemática & $\begin{array}{l}\text { Otras } \\
\text { disciplinas }\end{array}$ & Diferencia \\
\hline Que el profesor disfrute su tarea & $\begin{array}{l}\text { Ama su profesión, disfruta enseñar, ama lo que enseña, } \\
\text { transmite su amor o pasión por la materia, tiene } \\
\text { entusiasmo por enseñar }\end{array}$ & $43 / 118$ & $62 / 171$ & 0.0018 \\
\hline Que la materia le genere interés & $\begin{array}{l}\text { Desarrolla clases interesantes, hace la materia querible, } \\
\text { hace la materia atrapante, muestra la materia en forma } \\
\text { de desafío }\end{array}$ & $20 / 118$ & $35 / 171$ & -0.0352 \\
\hline Que el profesor esté motivado & Entusiasta / transmite entusiasmo, positivo, optimista & $16 / 118$ & $18 / 171$ & 0.0303 \\
\hline Que se capte su atención & $\begin{array}{l}\text { Sabe llegar al alumno, sabe captar la atención del } \\
\text { alumno, sabe mantener la atención del alumno }\end{array}$ & $9 / 118$ & $14 / 171$ & -0.0056 \\
\hline Que sea invitado a dar lo mejor de sí & $\begin{array}{l}\text { Da confianza, incentiva a sus alumnos, da aliento, incita } \\
\text { a que los alumnos puedan lograrlo, lleva a los alumnos } \\
\text { al límite de sus posibilidades de aprender, potencia la } \\
\text { autoestima }\end{array}$ & $13 / 118$ & $14 / 171$ & 0.0283 \\
\hline Que aprenda de manera significativa & $\begin{array}{l}\text { Hace perdurables los aprendizajes, motiva a los alumnos } \\
\text { para que estudien pensando, prepara para el mundo } \\
\text { exterior }\end{array}$ & $6 / 118$ & $15 / 171$ & -0.0369 \\
\hline $\begin{array}{l}\text { Que el profesor sea un profesional } \\
\text { comprometido }\end{array}$ & $\begin{array}{l}\text { Tiene profesionalismo, profesional, tiene participación } \\
\text { social }\end{array}$ & $6 / 118$ & $5 / 171$ & 0.0216 \\
\hline
\end{tabular}

En efecto, la máxima diferencia registrada se presenta en la familia de cualidades que aprenda de manera significativa, con un $3.69 \%$ a favor de las cualidades correspondientes a docentes de otras disciplinas. Le siguen que la materia le genere interés con 3.52\% también a favor de docentes de otras disciplinas y que el profesor esté motivado, con 3.03\% a favor de los profesores en Matemática. Esto recalca la importancia de la propia motivación del profesor en Matemática para motivar a su vez a sus alumnos en la clase.

En la dimensión 2, ¿Cómo preparan las clases? (tabla 5), se marcan algunas diferencias a favor de los profesores en Matemática en las familias denominadas con conocimiento (del 5.34\%) y con responsabilidad $(4.86 \%)$. De los profesores de otras disciplinas se distingue que planifican actividades vinculadas a la realidad $(5.75 \%)$ y que piensan la clase para contribuir al pensamiento de los alumnos (5.31\%). Le siguen las que ponderan que dicha preparación se realice de manera diferente $(3.93 \%)$ a favor de los docentes de otras disciplinas y con eficiencia $(3.71 \%)$ a favor de los docentes en Matemática. Parecieran ponderarse los profesores en Matemática con conocimiento, responsabilidad y eficiencia.

En la dimensión 3, ¿Cómo dirigen la clase? (tabla 6), se destaca, de los profesores de otras disciplinas, la familia promoviendo la participación estudiantil (con $4.04 \%$ a favor). En cuanto a los profesores en Matemática sobresalen explicando y enseñando bien $(8.64 \%$ a favor) y destinando tiempo para el aprendizaje (5.85\%). Es decir, se reconoce a los profesores en Matemática que saben brindar explicaciones oportunas y a su vez se muestran pacientes al interactuar con sus estudiantes en la clase.

\footnotetext{
${ }^{3}$ Además aparece la expresión general "motiva al alumno" no consignada en ninguna familia, con frecuencias 5/118 y 8/171 respectivamente.
} 


\section{Tabla 5. Comparación de cantidades por familias en la dimensión 2: ¿Cómo preparan las clases?}

\begin{tabular}{|c|c|c|c|c|}
\hline Familias $^{4}$ & Extractos de cualidades mencionadas & Matemática & $\begin{array}{l}\text { Otras } \\
\text { disciplinas }\end{array}$ & Diferencia \\
\hline Con conocimiento & $\begin{array}{l}\text { Inteligente, amplio en sus conocimientos, sabe la } \\
\text { materia, conoce, conoce de manera sólida, capacitado, } \\
\text { investigador, tiene todos los contenidos en la mente }\end{array}$ & $107 / 269$ & $135 / 392$ & 0.0534 \\
\hline Con responsabilidad & $\begin{array}{l}\text { Responsable, comprometido, trabajador, no falta a clase, } \\
\text { puntual, da todas las clases }\end{array}$ & $57 / 269$ & $64 / 392$ & 0.0486 \\
\hline Con dedicación & $\begin{array}{l}\text { Dedicado, dedicado al alumno, a la asignatura, aplicado, } \\
\text { dedicado a la enseñanza }\end{array}$ & $31 / 269$ & $37 / 392$ & 0.0208 \\
\hline Con eficiencia & Organizado, ordenado, prolijo, eficiente & $27 / 269$ & $26 / 392$ & 0.0371 \\
\hline $\begin{array}{l}\text { Procurando la accesibilidad del } \\
\text { conocimiento }\end{array}$ & $\begin{array}{l}\text { Sintético, da ejemplos claros, relaciona contenidos, } \\
\text { demuestra cada explicación, propone un resumen de cada } \\
\text { tema, busca distintas formas de dar clases }\end{array}$ & $20 / 269$ & $32 / 392$ & -0.0073 \\
\hline De manera diferente & $\begin{array}{l}\text { Creativo, enseña la asignatura de manera diferente, } \\
\text { busca siempre material nuevo, elabora trabajos prácticos } \\
\text { creativos, tiene estilo propio, tiene ideas originales }\end{array}$ & $10 / 269$ & $30 / 392$ & -0.0393 \\
\hline $\begin{array}{l}\text { Para contribuir al pensamiento de los } \\
\text { alumnos }\end{array}$ & $\begin{array}{l}\text { Preocupado en que sus alumnos aprendan, promueve } \\
\text { la reflexión, crítico, promueve apertura de pensamiento, } \\
\text { promueve conocer el porqué de las cosas, muestra } \\
\text { muchas verdades }\end{array}$ & $5 / 269$ & $28 / 392$ & -0.0531 \\
\hline $\begin{array}{l}\text { Con actividades vinculadas a la } \\
\text { realidad }\end{array}$ & $\begin{array}{l}\text { Da ejemplos cotidianos, hace transportar a los alumnos } \\
\text { al acontecimiento estudiado, aplica la teoría a la realidad, } \\
\text { propone trabajar mediante situaciones en contexto, } \\
\text { presenta temas de actualidad }\end{array}$ & $1 / 269$ & $24 / 392$ & -0.0575 \\
\hline $\begin{array}{l}\text { Con actividades y materiales para el } \\
\text { estudio }\end{array}$ & $\begin{array}{l}\text { Propone mucha práctica, prepara buen material de } \\
\text { estudio, aplica los conocimientos con muchos ejercicios, } \\
\text { promueve trabajo intensivo, propone trabajos prácticos } \\
\text { buenos }\end{array}$ & $10 / 269$ & $13 / 392$ & 0.0040 \\
\hline $\begin{array}{l}\text { Que el profesor sea un profesional } \\
\text { comprometido }\end{array}$ & $\begin{array}{l}\text { Tiene profesionalismo, profesional, tiene participación } \\
\text { social }\end{array}$ & $6 / 118$ & $5 / 171$ & 0.0216 \\
\hline
\end{tabular}

\footnotetext{
${ }^{4}$ Además aparecen la expresiones generales "sabe preparar la clase"; "tiene la clase bien preparada previamente" no consignadas en ninguna familia, con frecuencias 1/269; 0/269 y 12/392; 1/392 respectivamente.
} 


\section{Tabla 6. Comparación de cantidades por familias en la dimensión 3: ¿Cómo dirigen la clase?}

\begin{tabular}{|c|c|c|c|c|}
\hline Familias $^{5}$ & Extractos de cualidades mencionadas & Matemática & $\begin{array}{l}\text { Otras } \\
\text { disciplinas }\end{array}$ & Diferencia \\
\hline Explicando y enseñando bien & $\begin{array}{l}\text { Explica bien, desarrolla clases didácticas, desarrolla } \\
\text { clases muy buenas, tiene facilidad para transmitir sus } \\
\text { conocimientos }\end{array}$ & $244 / 654$ & $211 / 736$ & 0.0864 \\
\hline $\begin{array}{l}\text { Destinando tiempo para el } \\
\text { aprendizaje }\end{array}$ & $\begin{array}{l}\text { Paciente, explica muchas veces si es necesario, } \\
\text { predispuesto a enseñar, explica de diversas maneras, } \\
\text { enseña respetando los tiempos de aprendizaje, tiene } \\
\text { tiempo disponible para atender dudas, ayuda en las } \\
\text { dificultades si al alumno le cuesta }\end{array}$ & $160 / 654$ & $137 / 736$ & 0.0585 \\
\hline Siendo claros & $\begin{array}{l}\text { Claro, entendible, desarrollando clases comprensibles, } \\
\text { se esfuerza para promover el entendimiento, enseña } \\
\text { a entender lo complejo, los alumnos siguen sus } \\
\text { explicaciones }\end{array}$ & $88 / 654$ & $103 / 736$ & -0.0053 \\
\hline Siendo concisos & $\begin{array}{l}\text { Práctico / práctico al explicar, sencillo al explicar, preciso, } \\
\text { conciso, directo, explica sin dar tantas vueltas }\end{array}$ & $38 / 654$ & $57 / 736$ & -0.0163 \\
\hline Propiciando un ritmo adecuado & $\begin{array}{l}\text { Desarrolla clases dinámicas, maneja los diferentes } \\
\text { momentos de la clase }\end{array}$ & $32 / 654$ & $50 / 736$ & -0.0190 \\
\hline Expresándose de manera adecuada & $\begin{array}{l}\text { Sabe expresarse, expresivo, habla bien, tiene buena } \\
\text { oratoria, tiene lenguaje accesible, desenvuelto frente a la } \\
\text { clase, enseña a los alumnos a expresarse }\end{array}$ & $26 / 654$ & $49 / 736$ & -0.0268 \\
\hline Propiciando un clima agradable & $\begin{array}{l}\text { Desarrolla clases entretenidas, desarrolla clases } \\
\text { llevaderas, desarrolla clases amenas, desarrolla clases } \\
\text { como un juego, genera buen clima de trabajo }\end{array}$ & $25 / 654$ & $42 / 736$ & -0.0189 \\
\hline $\begin{array}{l}\text { Propiciando herramientas } \\
\text { transversales }\end{array}$ & $\begin{array}{l}\text { Enseña a razonar, enseña a estudiar, enseña cuestiones } \\
\text { de la vida, enseña a ser buenas personas, enseña a } \\
\text { estudiar no de memoria, transmite valores }\end{array}$ & $15 / 654$ & $34 / 736$ & -0.0233 \\
\hline $\begin{array}{l}\text { Promoviendo la participación } \\
\text { estudiantil }\end{array}$ & $\begin{array}{l}\text { Desarrolla clases participativas, desarrolla clases } \\
\text { interactivas, promueve el trabajo grupal, enseña a tener } \\
\text { opinión propia, promueve la ayuda entre los alumnos, } \\
\text { predispuesto a aceptar cambio de opiniones }\end{array}$ & $10 / 654$ & $41 / 736$ & -0.0404 \\
\hline Con presencia imponente & $\begin{array}{l}\text { Seguro, decidido, tiene presencia, contundente, } \\
\text { convincente }\end{array}$ & $13 / 654$ & $10 / 736$ & 0.0063 \\
\hline
\end{tabular}

\footnotetext{
${ }^{5}$ Además aparecen la expresiones generales "sabe manejar las clases"; "trabaja bien" no consignadas en ninguna familia, con frecuencias $3 / 654 ; 0 / 654$ y $1 / 736 ; 1 / 736$ respectivamente.
} 
Estos hallazgos confirman una vez más la trascendencia del momento de la clase, en tanto instancia privilegiada de construcción conjunta de sentidos, enfatizado aquí en las de Matemática. Como expresa Pennac (2008: 73): "Una sola certeza, la presencia de mis alumnos depende estrechamente de la mía: de mi presencia en la clase entera y en cada individuo en particular, de mi presencia también en mi materia, de mi presencia fisica, intelectual y mental, durante los cincuenta y cinco minutos que durará mi clase".

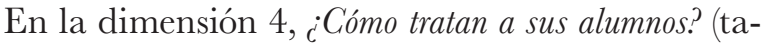
bla 7), de los profesores de otras disciplinas recordados como "buenos" se distinguen ligeramente las familias con compañerismo y demostrando que sus alumnos les importan $(3.5 \%$ ) y con alegría $(2.5 \%)$. En los profesores en Matemática se pondera especialmente que el trato sea con amabilidady gracia $(4.21 \%)$ y también valoran que en ocasiones sea de manera estricta $(2.56 \%)$ así como con generosidad (2.15\%).

En la dimensión 5, ¿Cómo evalúan? (tabla 8), una diferencia muy marcada de frecuencias se observa en la familia de cualidades siendo exigentes, con un 21.63\% a favor de los docentes en Matemática. Le sigue siendo equitativos, en la que las cualidades de docentes de disciplinas distintas a la Matemática superaron a los de ésta en un 9\%.

La marcada distinción de la familia siendo exigentes dentro de la Dimensión ¿Cómo evalúan? en los profesores en Matemática considerados memorables nos interpela profundamente. En primer término, no se puede obviar que los que lo están valorando favorablemente son justamente aspirantes a profesor en Matemática. Por otro lado, de acuerdo con hallazgos previos del equipo de investigación, la misma es especialmente reconocida cuando no se trata de una exigencia absurda o desmedida; por el contrario, comprende una exigencia basada en lo que el docente les brinda en cada encuentro a través de sus buenas explicaciones y con mucha paciencia (Sgreccia, Cirelli y Vital, 2015).
Seguimos advirtiendo que la terna de cualidades que caracteriza a cada docente memorable conjuga un interesante equilibrio del estilo del descrito por Flores et al. (2013: 88):

La enseñanza contiene muchas exigencias morales para los docentes: deben estar muy bien informados, pero ser respetuosos de quienes son ignorantes; ser amables y considerados, pero también estrictos y exigentes en ocasiones; estar libres de prejuicios, pero ser justos en su trato con los estudiantes; responder a las necesidades de cada alumno sin descuidar la clase en su conjunto; mantener el orden, pero permitiendo espontaneidad; ser optimistas y entusiastas aunque tengan dudas privadas; lidiar con lo inesperado.

\section{Con respecto a ciertas cualidades distinguidas}

Como se anunciara en el segundo apartado, en este plano de análisis se consideran las tres cualidades más recurrentes por dimensión. En la columna de "cualidades distinguidas" de la tabla 9 se las presenta de manera ordenada, consignándose entre paréntesis la frecuencia total de cada una. Seguidamente se muestra su ponderación de acuerdo con si los docentes son de Matemática o de otras disciplinas (hay 1782 y 2442 cualidades en total, respectivamente) y, como en tablas anteriores, se explicita la diferencia de tales frecuencias relativas. Desde esta perspectiva se efectúa, también en términos comparativos, una mirada a nivel puntual de las cualidades más recurrentes.

Luego de recorrer las cualidades distinguidas presentadas en la tabla 9 y focalizando en las tres más recurrentes (destacadas en Negrita), resulta que son:

- Para profesores de otras disciplinas: exigente $(6.51 \%$ del total de cualidades distinguidas mencionadas para estos docentes), explica bien $(5.16 \%)$ y buena persona $(4.01 \%)$. 
Tabla 7. Comparación de cantidades por familias en la dimensión 4: ¿Cómo tratan a sus alumnos?

\begin{tabular}{|c|c|c|c|c|}
\hline Familias $^{6}$ & Extractos de cualidades mencionadas & Matemática & $\begin{array}{l}\text { Otras } \\
\text { disciplinas }\end{array}$ & Diferencia \\
\hline Con amabilidad y gracia & $\begin{array}{l}\text { Simpático, amable, tiene buena onda, tiene buena } \\
\text { relación con los alumnos, atento, tiene buen carácter, } \\
\text { sociable, agradable, ameno }\end{array}$ & $115 / 589$ & $137 / 895$ & 0.0421 \\
\hline Con comprensión & $\begin{array}{l}\text { Comprensivo, consejero, escucha a los alumnos, } \\
\text { compasivo, contiene a los alumnos, se pone en el lugar } \\
\text { del alumno }\end{array}$ & $81 / 589$ & $138 / 895$ & -0.0167 \\
\hline Con calidad humana & $\begin{array}{l}\text { Buena, excelente persona, maestro, genio, completo, } \\
\text { groso / copado }\end{array}$ & $72 / 589$ & $104 / 895$ & 0.0060 \\
\hline $\begin{array}{l}\text { Con compañerismo y demostrando } \\
\text { que sus alumnos les importan }\end{array}$ & $\begin{array}{l}\text { Compañero, amigo, busca siempre una manera de ayudar, } \\
\text { atento a sus alumnos, cercano al alumno, comparte } \\
\text { momentos con sus alumnos, promueve la unión del curso, } \\
\text { defiende a sus alumnos, trata de igual a igual }\end{array}$ & $59 / 589$ & $121 / 895$ & -0.0350 \\
\hline Promoviendo respeto y disciplina & $\begin{array}{l}\text { Respetuoso, se hace respetar / genera respeto, } \\
\text { respetable, sabe ganarse el respeto de los alumnos, } \\
\text { promueve el respeto por los otros }\end{array}$ & $37 / 589$ & $65 / 895$ & -0.0098 \\
\hline Con alegría & $\begin{array}{l}\text { Divertido, alegre, gracioso, tiene buen humor, tiene } \\
\text { sentido del humor }\end{array}$ & $32 / 589$ & $71 / 895$ & -0.0250 \\
\hline De manera estricta & Estricto, recto, serio, tiene carácter & $46 / 589$ & $47 / 895$ & 0.0256 \\
\hline Con generosidad & $\begin{array}{l}\text { Dispuesto, solidario, predispuesto, generoso, bondadoso, } \\
\text { resuelve problemas del curso fuera de la clase, } \\
\text { predispuesto a ayudar siempre, tiene ganas de dar }\end{array}$ & $37 / 589$ & $37 / 895$ & 0.0215 \\
\hline Con apertura & $\begin{array}{l}\text { Humilde, tolerante, tiene buena comunicación con los } \\
\text { alumnos, habilita el diálogo, abierto, permite expresarse }\end{array}$ & $26 / 589$ & $27 / 895$ & 0.0139 \\
\hline Estableciendo límites claros & $\begin{array}{l}\text { Sabe manejar el curso, sabe poner orden, } \\
\text { promueve disciplina }\end{array}$ & $20 / 589$ & 19/895 & 0.0128 \\
\hline
\end{tabular}

\footnotetext{
${ }^{6}$ Además aparece la expresión general "tiene buen trato" no consignada en ninguna familia, con frecuencias 10/589 y 13/895 respectivamente.
} 


\begin{tabular}{|c|c|c|c|c|}
\hline Con afecto & $\begin{array}{l}\text { Cariñoso, dulce, ama a sus alumnos, amoroso, } \\
\text { afectuoso }\end{array}$ & 9/589 & $28 / 895$ & -0.0160 \\
\hline De manera personalizada & $\begin{array}{l}\text { Preocupado por cada alumno, interesado por los } \\
\text { alumnos, no hace preferencias entre los alumnos, } \\
\text { conoce a los alumnos, individualiza a cada alumno }\end{array}$ & $11 / 589$ & 22/895 & -0.0059 \\
\hline Con sinceridad & $\begin{array}{l}\text { Sincero, confiable, espontáneo, honesto, } \\
\text { consecuente con lo que dice, cumple con su } \\
\text { palabra }\end{array}$ & $15 / 589$ & $25 / 895$ & -0.0024 \\
\hline Siendo carismáticos & Carismático, admirable & $12 / 589$ & $25 / 895$ & -0.0075 \\
\hline Siendo flexibles & Flexible, no se enoja por cualquier cosa & $2 / 589$ & $12 / 895$ & -0.0100 \\
\hline Siendo humanos & Humano, humanitario, sensible & $5 / 589$ & $4 / 895$ & 0.0040 \\
\hline
\end{tabular}

Tabla 8. Comparación de cantidades por familias en la dimensión 5: ¿Cómo evalúan?

\begin{tabular}{|l|l|c|c|c|}
\hline \multicolumn{1}{|c|}{ Familias } & \multicolumn{1}{|c|}{ Extractos de cualidades mencionadas } & Matemática & $\begin{array}{c}\text { Otras } \\
\text { disciplinas }\end{array}$ & Diferencia \\
\hline Siendo exigentes & $\begin{array}{l}\text { Exigente, exigente con la materia, exigente en su límite, } \\
\text { exigente porque sabe que el alumno puede hacerlo mejor }\end{array}$ & $134 / 152$ & $165 / 248$ & 0.2163 \\
\hline Siendo equitativos & $\begin{array}{l}\text { Justo, objetivo, razonable, equitativo, imparcial para } \\
\text { evaluar }\end{array}$ & $9 / 152$ & $37 / 248$ & -0.0900 \\
\hline Dando oportunidades & $\begin{array}{l}\text { Da oportunidades para todos, da posibilidades de aprobar, } \\
\text { promueve que sus alumnos aprueben, saca a sus alumnos } \\
\text { adelante cuando les va mal }\end{array}$ & $2 / 152$ & $18 / 248$ & -0.0594 \\
\hline De manera continua & $\begin{array}{l}\text { Observador, transmite la necesidad del esfuerzo, califica } \\
\text { el cumplimiento de las tareas, corrige todo, exigente día a } \\
\text { día, efectúa seguimiento de sus alumnos }\end{array}$ & $3 / 152$ & $15 / 248$ & -0.0408 \\
\hline Mediante ciertos modos valorados & $\begin{array}{l}\text { Califica adecuadamente, evalúa siendo coherente con } \\
\text { lo que enseña, utiliza formas de evaluar valoradas a } \\
\text { posteriori por los alumnos }\end{array}$ & $4 / 152$ & $8 / 248$ & -0.0060 \\
\hline
\end{tabular}

\footnotetext{
${ }^{7}$ Además aparece la expresión general "evalúa bien” no consignada en ninguna familia, con frecuencias 0/152 y 5/248 respectivamente.
} 
- Para profesores en Matemática: explica bien $(9.93 \%$ del total de cualidades distinguidas mencionadas para estos docentes), exigente $(7.35 \%)$ y paciente $(6.9 \%)$.
Las mayores diferencias porcentuales a favor de las cualidades distinguidas en docentes de Matemática se observan en explica bien (4.77\%) y paciente (3.21\%). Las restantes diferencias son menores al 1\%.

\section{Tabla 9. Comparación de cantidades de ciertas cualidades distinguidas}

\begin{tabular}{|c|c|c|c|c|}
\hline Dimensión & Cualidades distinguidas & Matemática & $\begin{array}{c}\text { Otras } \\
\text { disciplinas }\end{array}$ & Diferencia \\
\hline \multirow{3}{*}{1} & Entusiasta / Transmite entusiasmo (18) & $11 / 1782$ & $7 / 2442$ & 0.0033 \\
\hline & Desarrolla clases interesantes (15) & 2/1782 & $13 / 2442$ & -0.0042 \\
\hline & Sabe llegar al alumno (13) & 8/1782 & $5 / 2442$ & 0.0025 \\
\hline \multirow{3}{*}{2} & Inteligente (92) & $46 / 1782$ & $46 / 2442$ & 0.0070 \\
\hline & Responsable (69) & $32 / 1782$ & $37 / 2442$ & 0.0028 \\
\hline & Dedicado (49) & 23/1782 & $26 / 2442$ & 0.0023 \\
\hline \multirow{3}{*}{3} & Explica bien (303) & $177 / 1782$ & $126 / 2442$ & 0.0477 \\
\hline & Paciente (213) & $123 / 1782$ & $90 / 2442$ & 0.0321 \\
\hline & Claro (139) & $59 / 1782$ & $80 / 2442$ & 0.0003 \\
\hline \multirow{3}{*}{4} & Buena persona (162) & $64 / 1782$ & $98 / 2442$ & -0.0042 \\
\hline & Comprensivo (157) & $64 / 1782$ & $93 / 2442$ & -0.0022 \\
\hline & Simpático (78) & $39 / 1782$ & $39 / 2442$ & 0.0059 \\
\hline \multirow{3}{*}{5} & Exigente (290) & $131 / 1782$ & $159 / 2442$ & 0.0084 \\
\hline & Justo (33) & $7 / 1782$ & $26 / 2442$ & -0.0067 \\
\hline & & 0/1782 & $14 / 2442$ & -0.0057 \\
\hline
\end{tabular}

\section{Discusión y conclusiones}

Para ambos grupos de docentes (de Matemática y de otras disciplinas), la mayor proporción de cualidades elegidas corresponde a las dimensiones Cómo dirigen la clase y Cómo tratan a sus alumnos, que refleja la observación de lo que sucede en el ámbito del aula y de cómo se desarrolla la actividad en el transcurso de la clase, así como la importancia atribuida a este momento. Cabe destacar que para los docentes de otras disciplinas el trato a los alumnos fue más valorado, con una diferencia del $20.6 \%$ con respecto a los docentes de Matemática, mientras que la forma en que éstos conducían las clases mostró una diferencia del $5.9 \%$. Se observa que los estudiantes apreciaron en sus profesores en Matemática la forma en la que dirigían las clases por sobre el trato brindado a los alumnos.

La labor dentro del recinto del aula puede o no evidenciar el trabajo previo y posterior de reflexión del docente, aspecto considerado en ambos grupos de profesores. La dimensión Cómo preparan las clases se ve disminuida con respecto a las dimensiones 
3 y 4, pero supera ampliamente a las dimensiones Qué motiva a un alumno y Cómo evalúan. Además, en estas últimas se advierte la mayor diferencia entre ambos grupos de docentes con respecto a la diversidad de cualidades mencionadas, con un $24 \%$ y $11 \%$ respectivamente a favor de los profesores de otras disciplinas. ¿Resultará entonces que los estudiantes, aspirantes a profesores en Matemática, ponderan la forma de motivar y evaluar en aquellas materias que no le son afines? Sin embargo, la exigencia de sus profesores en Matemática fue sumamente valorada por este grupo de estudiantes.

Por otro lado, estamos en condiciones de señalar que las cualidades que más valoran en los docentes memorables de otras disciplinas son: exigente-explica bien-buena persona, notando que se registra una variación con respecto a la terna sobresaliente entre todas las ternas obtenida en Sgreccia, Cirelli y Vital (2015): exigente-explica bien-paciente. ¿Estaría indicando que estos futuros profesores en Matemática necesitan en disciplinas no afines algo más que la paciencia del docente?, ¿̇requieren de ellos que sean buenas personas? En segundo término, cuando centramos la mirada en las cualidades ponderadas en los docentes memorables de Matemática, encontramos que la terna obtenida coincide con la terna de las ternas informada en Sgreccia, Cirelli y Vital (2015).

Con respecto a nuestro supuesto de partida: las seis cualidades sobresalientes (explica bien, exigente, paciente, buena persona, claro, comprensivo) de los profesores en general están aún más marcadas en los profesores en Matemática; a partir de la tabla 9, es posible reconocer indicios a favor en dos de ellas (explica bien con $4.77 \%$ y paciente con $3.21 \%$ ), ligeramente a favor en otras dos (exigente con $0.84 \%$ y claro con $0.03 \%$ ) y en contra en las dos restantes (comprensivo con $-0.22 \%$ y buena persona con $-0.42 \%)$.

Pareciera que los aspirantes a profesor en Matemática que reconocen, a su vez, a profesores en Matemática como docentes memorables de su escolaridad, distinguen sus explicaciones, su paciencia, su exigencia y su claridad. Dejan en segundo plano, en estos profesores, a las cualidades comprensivo y buena persona (iposiblemente por no haber sentido la necesidad de acudir a ellos en estos términos?).

Llegado este momento, nos permitimos integrar hallazgos del presente estudio con previos del equipo de investigación. También, nutrirlo con nuestro continuo intercambio en actividades de formación inicial y continua de profesores en Matemática, e intercalarlo con extractos de las Memorias Mal de Escuela, del escritor francés Daniel Pennac (2008), quien interpela sus experiencias escolares como un "mal" estudiante que dice haber sido.

Nos aventuramos en proponer que un buen profesor en Matemática es un profesor que configura su conocimiento profesional atendiendo a cuatro $P$ :

- Persistente: explica, exige...

La imagen del gesto que salva al ahogado, el puño que tira de ti hacia arriba a pesar de tu gesticulación suicida, esa ruda imagen de vida de una mano agarrando firmemente el cuello de una chaqueta es la primera que me viene a la cabeza cuando pienso en ello. En su presencia - en su materia- nacía yo para mí mismo: pero un yo matemático, si puedo decirlo así, un yo historiador, un yo filósofo, un yo que, durante una hora, me olvidaba un poco, me ponía entre paréntesis, me libraba del yo que, hasta el encuentro con aquellos maestros, me había impedido sentirme realmente allí (Pennac, 2008: 149).

- Paciente: explica de diversas maneras, se pone en el lugar del otro...

Tomaban en consideración tanto a sus buenos como a sus malos alumnos, y sabían reanimar en los segundos el deseo de comprender. Acompañaban paso a paso nuestros esfuerzos, se alegraban de nuestros progresos, no se impacientaban por nuestras lentitudes, nunca consideraban nuestros fracasos como una 
injuria personal y se mostraban con nosotros de una exigencia tanto más rigurosa cuanto estaba basada en la calidad, la constancia y la generosidad de su propio trabajo (Pennac, 2008: 149).

Un docente paciente es, por sobre todas las cosas, un docente que cree fehacientemente en sus alumnos; los reconoce y respeta como tales. "Dedicó aquel año a sacarnos del abismo de nuestra ignorancia... se maravillaba siempre de lo que sabíamos a pesar de todo. -Creéis que no sabéis nada, pero os equivocáis, os equivocáis, ¡sabéis muchísimas cosas! Mira, Pennacchioni, ¿sabías que lo sabías?" (Pennac, 2008: 148).

- Presente: se puede contar con él, prepara sus clases...

La presencia del profesor que habita plenamente su clase es perceptible de inmediato. Los alumnos la sienten desde el primer minuto del año, todos lo hemos experimentado: el profesor acaba de entrar, está absolutamente allí, se advierte por su modo de mirar, de saludar a sus alumnos, de sentarse, de tomar posesión de la mesa. No se ha dispersado por temor a sus reacciones, no se ha encogido sobre sí mismo, no, él va a lo suyo, de buenas a primeras, está presente, distingue cada rostro, para él la clase existe de inmediato (Pennac, 2008: 75).

- (Con) Pasión: le gusta lo que hace, transmite entusiasmo...

Desde sus primeras palabras, nos adentrábamos en las matemáticas. ¿Con qué estaba hecha aquella hora que tanto nos retenía? Esencialmente con la materia que el señor Bal enseñaba y que parecía habitarle, lo que le convertía en un ser curiosamente vivo, tranquilo y bueno. Extraña bondad, nacida del propio conocimiento, deseo natural de compartir con nosotros la "materia" que arrobaba su espíritu y de la que no podía concebir que nos resultara repulsiva, o sencillamente ajena. Bal estaba amasado con su materia y sus alumnos... tan convincente era su gozo al enseñar (Pennac, 2008: 147).

Esta pasión la puede tener alguien que domina fuertemente su materia. Decimos que un profesor en Matemática con esta característica tiene un conocimiento matemático para la enseñanza (Ball et al., 2008) altamente desarrollado.

Nos daban clases tan memorables como el teorema, el tratado de paz o la idea fundamental, que aquel día eran el tema. Enseñándolo, creaban el acontecimiento. Y otra cosa, me parece que tenían cierto estilo. Eran artistas en la transmisión de su materia. Sus clases eran actos de comunicación, claro está, pero de un saber dominado hasta el punto de pasar casi por creación espontánea. Su facilidad convertía cada hora en un acontecimiento que podíamos recordar como tal (Pennac, 2008: 150).

Un docente de las cuatro $P$ es, sin lugar a dudas, un docente comprometido con su trabajo a quien no le da lo mismo realizar este trabajo que cualquier otro. Suele darse que muchas veces nos pasamos el tiempo esperando el momento o las circunstancias oportunas (Terigi, 2010) quejándonos que no fuimos preparados para actuar en el contexto en que nos toca actuar, como si un cambio en ese contexto ya fuera garantía por sí mismo de la calidad educativa.

Los profesores que me salvaron - y que hicieron de mí un profesor - no estaban formados para hacerlo. No se preocuparon de los orígenes de mi incapacidad escolar. No perdieron el tiempo buscando sus causas ni tampoco sermoneándome. Eran adultos enfrentados a adolescentes en peligro. Se dijeron que era urgente. Se zambulleron. No lograron atraparme. Se zambulleron de nuevo, día tras día, más y más... Y acabaron sacándome de allí. Y a muchos 
otros conmigo. Literalmente, nos repescaron. Les debemos la vida (Pennac, 2008: 23).

También, como docentes deberíamos ser conscientes de las marcas, ¡de por vida!, que dejamos en nuestros alumnos en general y de la exponencial trascendencia que esto tiene si nuestros alumnos son, a su vez, futuros docentes. "Solo sé que los tres estaban poseídos por la pasión comunicativa de su materia. Armados con esa pasión, vinieron a buscarme al fondo de mi desaliento y solo me soltaron una vez que tuve ambos pies sólidamente puestos en sus clases, que resultaron ser la antecámara de mi vida" (Pennac, 2008: 149).

Para finalizar deseamos reconocer el potencial que tiene este tipo de estudios para realizar aportes concretos en la formación, tanto inicial como continua, de profesores en Matemática. "Si, además del de los maestros célebres, esa antología ofreciera el retrato del profesor inolvidable que casi todos nosotros hemos conocido una vez al menos en nuestra escolaridad, tal vez obtuviéramos ciertas luces sobre las cualidades necesarias para la práctica de ese extraño oficio" (Pennac, 2008: 152).

En el PM el tema de los docentes memorables en la biografía escolar se ha constituido en un eje de trabajo articulador en la carrera que se ha venido robusteciendo a través de los años a partir de las vinculaciones entre investigación y docencia.

En el anexo 1 compartimos, a modo de ejemplo, las consignas de trabajo que se proponen a los estudiantes durante el trayecto de la práctica docente. Tienen la intención de despertar y fortalecer la conciencia acerca de la complejidad de la labor docente así como de resaltar la importancia de desarrollar conocimientos y actitudes necesarios para el buen ejercicio de la profesión.

Coincidimos con Davini (2015) en que la formación inicial no es una "empresa de bajo impacto" sino que es la encargada de generar los cimientos de la futura acción profesional. Es el ámbito propicio para promover cambios en aquellos modelos docentes interiorizados y fuertemente enraizados durante los años escolares que, de no ser desnaturalizados, obturan la posibilidad de construcción de nuevos conocimientos acerca de las prácticas docentes (Edelstein, 2015). Comprometidas con estas ideas y a fin de colaborar en la configuración de nuevos esquemas de acción por parte de los estudiantes, continuaremos indagando acerca del aporte de la biografía escolar en las instancias de formación inicial de Profesores en Matemática. 


\section{Referencias}

Alliaud, Andrea (2004), "La experiencia escolar de maestros 'inexpertos'. Biografías, trayectorias y práctica profesional", en Revista Iberoamericana de Educación, vol. 34, núm. 3, pp. 1-12.

Álvarez, Zelmira, Luis Porta y María Cristina Sarasa (2011), "Buenas prácticas docentes en la formación del profesorado: Relatos y modelos entramados", en Revista de Currículum y Formación del Profesorado, vol. 15, núm. 1, pp. 241-252.

Bain, Ken (2007), Quéhacen los mejores profesores universitarios, Valencia, Universitat de València.

Ball, Deborah, Mark Thames y Geoffrey Phelps (2008), "Content knowledge for teaching. What makes it special?", en Fournal of Teacher Education, vol. 59, núm. 5, pp. 389-407.

Cabrera, Isaac (2009), "El análisis de contenido en la investigación educativa: propuesta de fases y procedimientos para la etapa de evaluación de la información", en Pedagogía Universitaria, vol. 14, núm. 3, pp. 71-93.

Davini, María Cristina (2015), La formación en la práctica docente, Buenos Aires, Paidós.

De Laurentis, Claudia (2014), "Docentes memorables en la UNMDP: metáforas que construyen identidad", en Revista Entramados, núm. 1, pp. 99-107.

Edelstein, Gloria (2015), "La enseñanza en la formación para la práctica”, en Educación, Formación e Investigación, vol. 1, núm. 1, pp. 1-11.

Flores, Graciela, Zelmira Álvarez y Luis Porta (2013), "La buena enseñanza en la educación superior: profesores memorables y valores memorables", en Revista Magistro, vol. 7, núm. 13, pp. 81-108.

Hernández, Roberto, Carlos Fernández y Pilar Baptista (2006), Metodología de la investigación (4ª ed.), México, Mc Graw Hill.

Jackson, Philip (1999), Enseñanzas implícitas, Buenos Aires, Amorrortu.

Malikow, Max (2005-2006), "Effective teacher study", en National Forum of Teacher Education Fournal-Electronic, vol. 16, núm. 3e, pp. 1-9.

Osorio, Marta (2012), "Profesores universitarios memorables, a través de sus relatos autobiográficos", en Revista Hallazgos, vol. 9, núm. 18, pp. 97-117.

Pennac, David (2008), Mal de escuela, Barcelona, Mondadori.

Sarasa, M.C. (2014), "Experiencias de enseñanza y de aprendizaje mediante la investigación biográfica y la indagación narrativa", en Revista de Educación, 5(7), pp. 157-170, <http://fh.mdp.edu.ar/revistas/index. php/r_educ/article/view/986/1027>.

Sgreccia, Natalia y Mariela Cirelli (2015), "Cualidades de docentes memorables destacadas por aspirantes a profesor en Matemática", en Revista de Currículum y Formación del Profesorado, vol. 19, núm. 2, pp. 333-349.

Sgreccia, Natalia, Mariela Cirelli y María Beatriz Vital (2016), “¿Qué cualidades de aquellos buenos docentes reconozco en mí? Un estudio con egresadas del Profesorado en Matemática de la Universidad Nacional de Rosario", en Revista de Educación, vol. 7, núm. 9, pp. 297-315.

Sgreccia, Natalia, Mariela Cirelli y María Beatriz Vital (2015), "Docentes memorables destacados por ingresantes al Profesorado en Matemática. Hacia una tipología de análisis", en VIII Fornadas Nacionales y I Congreso Internacional sobre la Formación del Profesorado, Mar del Plata, Universidad Nacional de Mar del Plata.

Terigi, Flavia (2010), "Las cronologías de aprendizaje: un concepto para pensar las trayectorias escolares", en Fornada de Apertura Ciclo Lectivo 2010, Santa Rosa, Ministerio de Educación.

Vital, María Beatriz, Natalia Sgreccia y Mariela Cirelli (2015), "Aportes de la biografía escolar para las prácticas de la enseñanza de la Matemática", en $I V$ Fornada de Experiencias Innovadoras en Educación en la FCEIA, Rosario, Universidad Nacional de Rosario 


\section{Anexo 1}

\section{Práctica de la Enseñanza I}

Luego de un primer procesamiento de los datos vertidos en la Ficha que se muestra en la Fig. 1, para el año de cursado respectivo (se ejemplifica con 2017), en sucesivas clases durante el primer mes de cursado se plantea:

Análisis de las características generales de la muestra en estudio (grupal)

Pensar, discutir en el grupo, acordar y responder:

- ¿Por qué creen que hay más docentes femeninos?

- ¿Por qué creen que hay más docentes de Matemática?

- ¿A qué creen que responde el hecho de que la mayoría de los docentes recordados correspondan al ciclo orientado de la enseñanza secundaria? (años $3^{\circ}, 4^{\circ}$ y $5^{\circ}$ )

Análisis de las cualidades (grupal)

- Desde "Claro" hasta "Justo" (inclusive): pensar, discutir en el grupo, acordar y explicitar cuál es la importancia que reviste, en el ejercicio de la docencia, cada una de estas cualidades, emergidas de la encuesta)

- Para las cualidades: Se preocupa porque los alumnos entiendan; Inteligente; Mucho conocimiento disciplinar; Exigente; Perseverante; Versátil; Clases participativas; Clases divertidas: analizar cada una y explicitar si la consideran menos importante que las anteriores. En caso afirmativo, explicar bien por qué. En caso negativo, tratar de interpretar y explicar por qué creen que fue poco mencionada por los alumnos encuestados.

Análisis de la naturaleza de las cualidades positivas de los "Buenos Docentes" (grupal)

- Analizar, debatiendo en el grupo, para cada una de las cualidades indicadas en la tabla, con cuál de los siguientes aspectos está más fuertemente vinculada (o de cuál depende en forma más marcada):

1) Formación disciplinar (en la materia o disciplina que se enseña)

2) Formación pedagógica

3) Personalidad del docente

4) Actitud o voluntad del docente

- Completar la siguiente tabla para mostrar la conclusión de ese análisis.

\begin{tabular}{|l|l|}
\hline Cualidad & No de aspecto con el cual se vincula más fuertemente \\
\hline Claro & \\
\hline Compañero & \\
\hline
\end{tabular}

Ideas vertidas en la interpretación de los resultados de la encuesta "Buenos Docentes" (grupal)

- Buscar, en las respuestas a las preguntas de los trabajos anteriores, ideas comunes vertidas por los diferentes grupos, explicitar esas ideas asignándoles una descripción breve que sintetice lo expresado e indicar, en cada caso, cuál o cuáles fueron los grupos que hicieron referencia a esa idea (como se hizo en clase). Indicar también, si hay, alguna idea que discrepe totalmente de las comunes.

- Efectuar el mismo análisis que en el ítem anterior para las cualidades Inteligente, Mucho conocimiento disciplinar y Exigente

Yo, futuro docente (individual)

- De las cualidades de los Buenos Docentes surgidas de la encuesta realizada en este curso el primer día de clases, elegir y mencionar a lo sumo cinco que te gustaría tener como docente, explicando el porqué de tu elección.

- De las cualidades elegidas:

¿Cuáles crees que ya has desarrollado?

¿Cuáles crees que puedes desarrollar a lo largo de la carrera del Profesorado?

¿Qué crees que debería enseñarse en la carrera para que logres alcanzar o mejorar esas cualidades? 


\section{Práctica de la Enseñanza II}

El primer día de clase se llevan las fichas con datos de docentes memorables (fig. 1), completadas durante los últimos años, separadas en sobres según cohorte. Cada estudiante extrae la ficha que completó cuando cursó Práctica de la Enseñanza I (la reconoce él mismo, pues había sido completada de manera anónima). A continuación se solicita que:

- Cada uno lea en voz alta lo escrito.

- Intenten reconocer los sentidos de esas palabras, es decir, qué habrán querido decir con esas palabras.

- Comenten si hoy volverían a elegir a esos profesores con esas cualidades y por qué.

- Cuáles cualidades reconocen hoy en sí mismos como profesores en formación.

- Debido a qué creen que se las han ido incorporando.

- Cuánto tuvieron que ver los docentes memorables reconocidos en Práctica de la Enseñanza I.

- Cuánto, la carrera Profesorado en Matemática.

- Si un alumno ingresante al Profesorado en Matemática reconocería a alguno de ustedes, qué cualidades creen que destacarían (escribirlas).

- Compartan las cualidades escritas, con sus sentidos y reconozcan el trabajo que les demandan poseerlas.

De tarea deben realizar un Informe individual destacando:

- ¿Qué cualidades son imprescindibles para un buen profesor, en general y en Matemática en particular?

- ¿Cómo consideran que se desarrollan?

En la siguiente clase los docentes realizan una devolución de cada producción en el grupo-clase, a modo de retroalimentación, y se comparten los aportes de Bain (2007) así como algunos hallazgos de nuestro equipo de investigación.

\section{Residencia}

En el primer día de clase se solicita a los residentes:

Responder, de manera escrita e individual, las siguientes preguntas:

1. ¿Cómo te ves como profesor en Matemática? Enumerar características e indicar a qué crees que se debe que las tienes desarrolladas.

2. ¿Has tenido docentes en tu escolaridad (preescolar, primaria, secundaria, universitaria) con tales características? ¿O todo lo contrario? Indicar el nivel educativo, la asignatura y una breve descripción.

3. ¿Qué cualidades te gustaría tener como futuro profesor en Matemática que en la actualidad todavía no posees? ¿Por qué te parece importante incorporarlas?

Se trabaja un par de lecturas (Alliaud, 2004; Jackson, 1999) y se proyectan videos de tres clases de Matemática de nivel secundario, con la intención de reconocer elementos constituyentes del accionar del profesor y establecer relaciones con las propias experiencias de los residentes. También se ponen a disposición los listados de cualidades indicadas por los estudiantes en Práctica de la Enseñanza I durante los últimos 10 años. A partir del trabajo inicial y de estos materiales, se solicita:

Escribir un informe que contemple, en un texto único, los siguientes puntos, que se ofrecen a modo de guía:

- Cualidades más valoradas (entre tres y cinco) por los estudiantes del Profesorado en Matemática al ingresar a la carrera. Para cada cualidad, intentar dar una interpretación de la misma.

- Coincidencias y diferencias con las cualidades valoradas por los residentes. Si se detectaran diferencias, intentar dilucidar a qué se debe.

- ¿ ¿Hay cualidades, que ustedes valoran hoy, que no aparecen en las encuestas? De ser así, ¿a qué creen que se debe?

- ¿La valoración de cualidades de un docente ha variado a lo largo de los años analizados? De ser así, ¿qué ha cambiado?, ¿a qué creen que se debe?

- ¿Hay algunas cualidades que les han llamado la atención? ¿Por qué? 
- ¿Recuerdan ustedes alguna cualidad que valoraban al inicio de la carrera y ahora ya no? ¿A qué creen que se debe?

- ¿Creen que la reflexión sobre la biografía escolar es importante para la formación docente? De ser importante, ¿en cuál o cuáles de las etapas de la misma lo es?, ¿por qué?

- Conclusiones y reflexión final.

Para finalizar, entre los ejes de análisis para el ensayo monográfico de cierre del trayecto como residente, se propone consignar: ¿De qué modo consideras que tu biografía escolar condicionó/posibilitó tus prácticas docentes en tu experiencia como residente? 